polypeptides et les acides aminés. On précipite par $1 \mathrm{~cm}^{3}$ d'acide phosphotungstique (réactif de Folin), et on centrifuge le précipité. On reprend le culot, comme dans l'opération précédente ; on le dilue à $10 \mathrm{~cm}^{3}$, on reprend $9 \mathrm{~cm}^{3}$ (correspondant à $3 \mathrm{~cm}^{3}$ de lait) et on pratique la réaction des phénols comme ci-dessus.

\title{
Exemple numérique
}

Densité optique lue au Bonet-Maury (Ecran 15) $=68,0$ 5,2 (témoin) $=62,8$ correspond, d'après le grâphique de référence, à $0 \mathrm{mgr}$. 46 de tyrosine, d'où :

$\frac{0,46 \times 1.000}{3}=153$ milligrammes de tyrosine par litre de lait, ou : $153 \times 16,3=2$ gr. 49 de polypeptides par litre de lait, exprimé en caséine.

\section{Conclusions}

Nous avons donné, dans un précédent travail, un procédé colorimétrique basé sur le pouvoir chromogène des protides traité par le réactif des phénols, pour mesurer les protides totaux du lait.

Nous indiquons ici, comment on peut doser séparément la caséine, la lactalbumine et les polypeptides d'après ce même principe.

\section{BIBLIOGRAPHIE}

Folx et Denis. A colorimétric méthod for the détermination of phénol (and phénol dérivates) in urine. $J$. Biol. Chem., 1915, 22, 305.

R. Goiffon et Spaey. Mesure de l'Index. Tyrosine des polypeptides sériques. Bull. de la Soc. Chimie Biol., décembre 1934, p. 16.

Etienne Gorffon. Dosage pratique des protides du lait par la mesure colorimétrique de la tyrosine et du tryptophane. Le Lait, 1948, t. XXVIII, p. 449 .

\section{SÉCRÉTION LACTÉE HUMAINE ET ALIMENTATION HYDRIQUE (1)}

par

MaRCel LELONG, Mme F. ALISON et J. VINCENEUX

L'opinion qu'il est possible de modifier, la sécrétion lactée quantitativement et qualitativement par des variations quantitatives et qualitatives de l'alimentation est un préjugé encore fortement enraciné. E. Lambling recommandait d'ajouter, à la ration de la nourrice, un surplus de glucides et de lipides dans l'espoir

(1) Archives françaises de Pédiatrie, 1948, 5, no 4, 337. 
d'obtenir un lait plus abondant et plus butyreux et la coutume est encore vivace de recommander aux femmes allaitantes, du lait, du beurre, des aliments amylacés tels que pommes de terre, pain, riz, pois verts et sees, lentilles et autres légumes en grain "qui excitent la sécrétion lactée " (A. Gautier) (1).

En physiologie animale, cependant, il y a déjà longtemps que Porcher a montré que, dès lors que l'alimentation de base est assurée, on ne peut mettre en évidence une influence directe de l'alimentation sur la sécrétion lactée, aussi bien en ce qui concerne son volume global que sa composition, les vitamines étant, bien entendu, exclues, et d'une façon générale les principes mineurs que l'organisme ne sait fabriquer et doit nécessairement tirer du milieu extérieur. "Dès l'instant où vous aurez donné à l'animal tout ce qui est nécessaire pour s'entretenir et satisfaire aux besoins de sa sécrétion lactée, il deviendra inutile de forcer les doses de tel ou tel principe alimentaire dans le but de voir la proportion du principe correspondant s'élever dans le lait (2). ”

Cette loi, bien établie en expérimentation vétérinaire, est, a priori, transposable en physiologie humaine. Mais, pour la vérifier, l'expérimentation est ici plus délicate, souvent irréalisable, et en tous cas risque souvent d'être gênée par l'interférence de facteurs psychiques. Toutefois, grâce aux conditions privilégiées d'observation dont nous profitons au Centre de collecte de lait de femme de l'hôpital Saint-Vincent-de-Paul, nous avons essayé d'aborder ce problème. A cause de sa complexité, il nous a paru nécessaire de le diviser, et, dans cette première étude, nous nous limitons à son aspect le plus simple : le rôle de l'alimentation hydrique. Il est en effet relativement facile, en maintenant fixes les autres constituants de la ration alimentaire, de faire varier seulement les boissons ingérées. Dans ce domaine règnent d'ailleurs assez d'idées erronées, pour qu'un pareil travail présente quelque intérêt.

Les nourrices qui se sont prêtées à notre observation ont été soigneusement choisies. Ce sont des donneuses sédentaires, soumises au régime de l'internat. Leur alimentation a pu être rigoureusement contrôlée. Rompues à la pratique de l'expression manuelle du sein, elles étaient capables d'extraire la totalité de leur sécrétion mammaire quotidienne : pendant toute la durée de l'expérience, nos examens ont porté sur la sécrétion lactée des 24 heures, recueillie intégralement.

Quoique plusieurs nourrices se soient pliées aux rigueurs de cette expérimentation, nous ne rapportons volontairement que deux

(1) Armand Gautier. L'alimentation et les régimes. Masson, édit., 1908, p. 530. Vildermann. Archives de Médecine des Enfants, juillet 1901, p. 391.

(2) Ch. Porcher. In Traité de Physiologie de G. Roger et Léon Binet, t. XI, p. 352. 
séries d'épreuves réalisées chez une seule d'entre elles, parce que nous avons eu, à maintes reprises, la preuve de l'honnêteté, de la docilité et même du dévouement de cette donneuse : elle avait compris la portée de l'effort qui lui était demandé et exécutait nos prescriptions avec une parfaite exactitude.

Cette femme, - Mme L..., - âgée de 23 ans, était bien portante et n'avait aucun antécédent pathologique. Admise au Centre de donneuses de lait en juillet 1946, elle s'est soumise à notre étude au début d'octobre. Elle avait donc eu le temps de s'habituer au service ; elle y menait une vie calme et reposante, son état psychique était normal. Son enfant, le premier, de santé florissante, était âgé de 4 mois et demi ; elle donnait un lait "mûr ", dont le volume quotidien oscillait entre $900 \mathrm{~cm}^{3}$ et $1.100 \mathrm{~cm}^{3}$.

Son alimentàtion, pesée chaque jour, était maintenue au taux moyen de 4.000 ealories, ses constituants étant physiologiquement équilibrés.

Le volume des liquides ingérés (lait, tilleul, eau, bière), était mesuré chaque jour. Il n'a pas été tenu ữn compte séparé de l'eau de constitution des aliments ; étant donné le régime suivi, nous l'avons considérée comme constante et l'avons négligée ; en novembre et décembre, la consommation en légumes aqueux et en fruits frais a été pratiquement nulle.

Les urines ont été recueillies chaque jour.

Enfin, le volume du lait secrété a été chaque jour mesuré dans une éprouvette graduée. Le résultat des 6 ou 7 traites journalières, faites à intervalles réguliers, a été mélangé. Les dosages, portant sur les matières grasses, le lactose, les protides, l'extrait see dégraissé, ont été effectués sur un échantillon prélevé sur le mélange des 24 heures.

Nous avons ainsi tracé les courbes quotidiennes des boissons ingérées, des urines éliminées et du lait sécrété.

Les résultats suivants ont été obtenus :

\section{Première expérience}

\section{- du 23 au 27 novembre 1946 : liberté des boissons.}

$\mathrm{M}^{\mathrm{me}} \mathrm{L}$. boit à sa soif, mais note fidèlement les quantités ingérées. Celles-ci s'inscrivent entre 2.000 et $2.600 \mathrm{~cm}^{3}$ par jour.

L'élimination urinaire quotidienne est entre $2.800 \mathrm{~cm}^{3}$ et $3.000 \mathrm{~cm}^{3}$.

La production de lait varie peu d'un jour à l'autre : de 940 à $1.060 \mathrm{~cm}^{3}$. Le lait a la composition moyenne suivante, par litre :

Matières grasses $\ldots \ldots \ldots \ldots \ldots \ldots \ldots \ldots \ldots \ldots$

37,87 Grammes

Lactose . . . . . . . . . . . . . . .

72,48

Protides ........................ 16,50

Extrait see dégraissé $\ldots \ldots \ldots \ldots \ldots \ldots \ldots \ldots . \quad 89,83$ 
- du 28 novembre au 8 décembre (1 er au 11 jo jour de l'expérience) : restriction des boissons.

Les boissons sont réduites progressivement et tombent à $800 \mathrm{~cm}^{3}$ par jour pendant les trois derniers jours de la restriction.

Pendant cette période, la diurèse descend peu à peu à $500 \mathrm{~cm}^{3}$ par jour.

La production quotidienne de lait reste à peu près fixe, entre $990 \mathrm{~cm}^{3}$ et $1.100 \mathrm{~cm}^{3}$, ses oscillations restant dans les limites physiologiques.

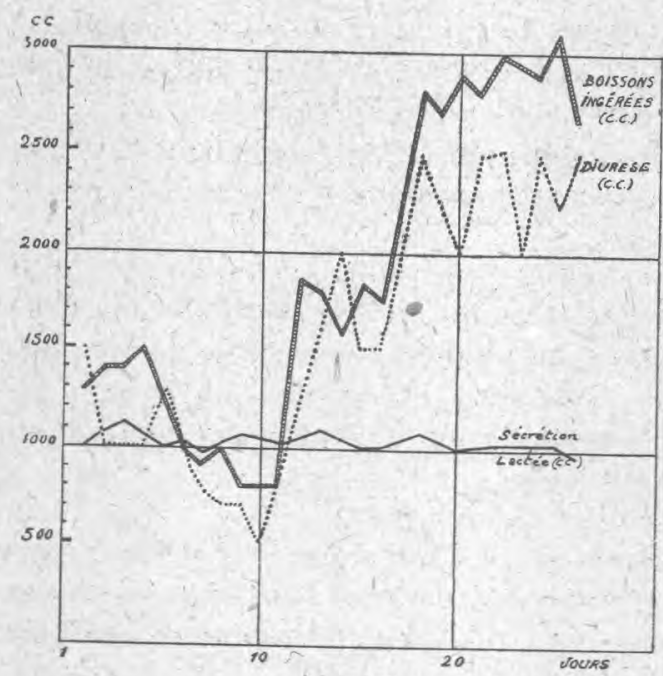

Frg. 1

1re expérience. - La diurèse varie parallèlement. à l'ingestion des boissons. La sécrétion lactée reste inchangée.

Le lait secrété a la composition suivante, le dernier jour de la restriction :

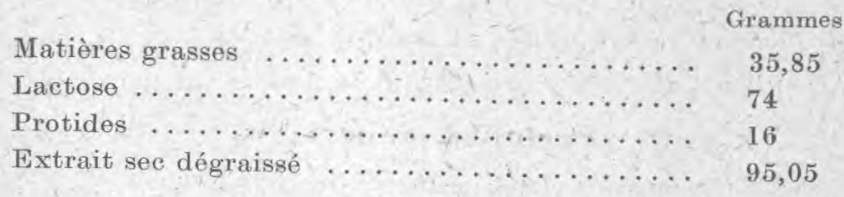

- du 9 au 23 décembre $\left(12^{\mathrm{e}}\right.$ au $26^{\mathrm{e}}$ jour de l'expérience) : augmentation des boissons.

Mme L. s'efforce de boire le plus possible, au delà de sa soif. Elle ingère chaque jour, entre $2.800 \mathrm{~cm}^{3}$ et $3.100 \mathrm{~cm}^{3}$ de liquides, pendant les neuf derniers jours de l'épreuve.

La diurèse remonte peu à peu à $2.500 \mathrm{~cm}^{3}$.

La production lactée reste invariable : entre 990 et $1.085 \mathrm{~cm}^{3}$.

La composition du lait, le 23 décembre, est la suivante : 
Grammes

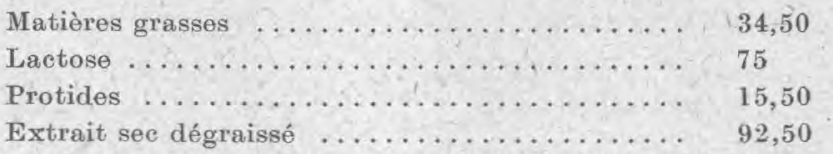

L'excès des liquides ingérés a été éliminé par le rein ; la sécrétion lactée n'a été modifiée, ni dans son volume global, ni dans sa composition centésimale.

\section{Deuxième expérience}

Le processus expérimental est le même que précédemment. Mais la nourrice est pesée un jour sur deux et l'expérience est poursuivie avec plus de ténacité, pendant 45 jours. Cet essai, par sa rigueur et sa durée, nous paraît a voir un intérêt exceptionnel.

- du 21 janvier au 15 février 1947 (10r au 26e jour de l'expérience) : restriction des boissons.

Cette restriction se fait par étapes. La quantité de liquide

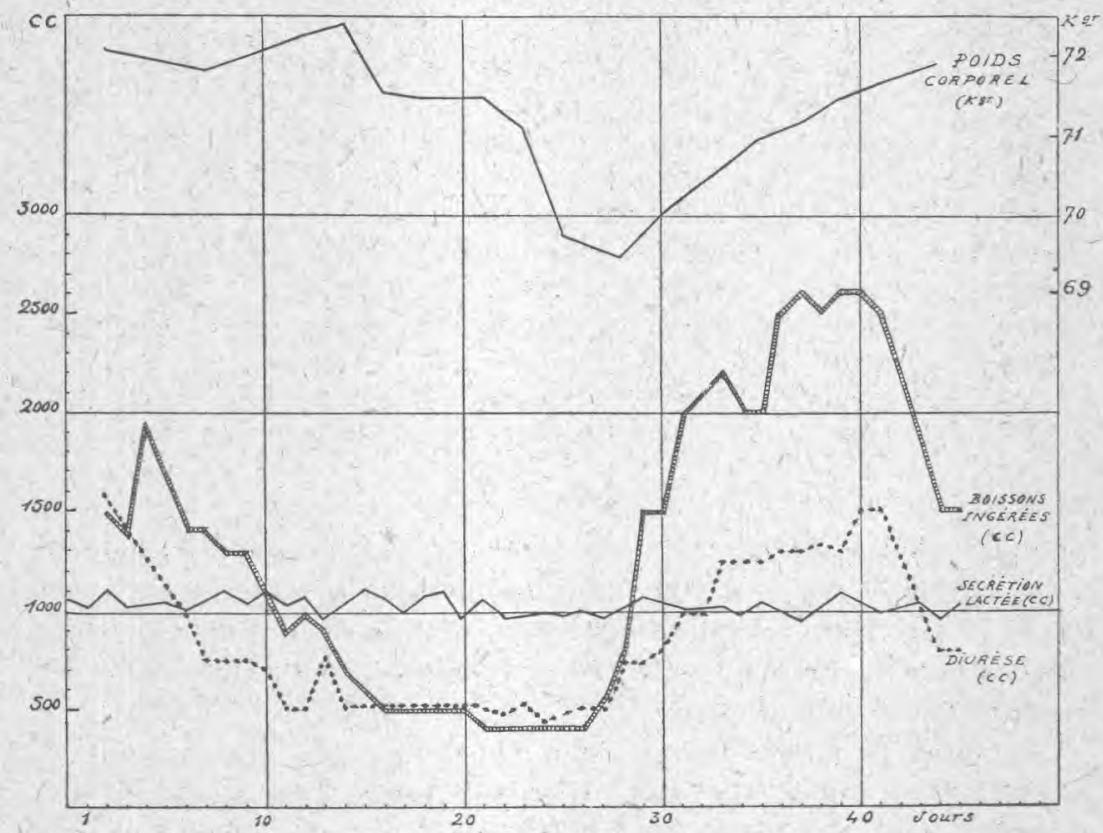

Fig. 2

2e expérience. - La restriction des ingestions liquidiennes, très poussée, amène une diminution de la diurèse ainsi qu'une chute du poids de la nourrice. Ensuite l'ingestion dè quantités croissantes de liquides entraîne une augmentation de la diurèse et une reprise du poids. La séerétion lactée ne vario pas. 
M. LELONG, Mme F. ALISON ET J. VINCENEUX. - SÉCRÉTION

ITe EXPLRIENCE

\begin{tabular}{|c|c|c|c|c|c|c|c|}
\hline Dates & $\begin{array}{c}\text { Boissons } \\
\text { ingérées } \\
\left(\mathrm{em}^{3}\right)\end{array}$ & $\begin{array}{c}\text { Diurèse } \\
\left(\mathrm{em}^{3}\right)\end{array}$ & $\begin{array}{c}\text { Sécrétion } \\
\text { lactée }\left(\mathrm{cm}^{3}\right)\end{array}$ & $\begin{array}{c}\text { Extrait sec } \\
\text { dégraissé } \\
\left(\text { gr. } \%{ }^{\circ}\right)\end{array}$ & $\begin{array}{l}\text { Protides } \\
\left(\text { gr. } \%{ }^{\circ}\right)\end{array}$ & $\begin{array}{l}\text { Lipides } \\
\left(\text { gr. } \%{ }^{\circ}\right)\end{array}$ & $\begin{array}{l}\text { Laetose } \\
\left(\text { gr. \% }{ }^{0}\right)\end{array}$ \\
\hline $28 \mathrm{XI}$ & 1300 & 1500 & 995 & - & - & - & - \\
\hline 29 & 1400 & 1000 & 1075 & 92,40 & 16,50 & 39,50 & 72,50 \\
\hline 30 & 1400 & 1000 & 1110 & - & - & - & - \\
\hline $1 \mathrm{XII}$ & 1500 & 1000 & 1060 & 91,10 & 17,50 & 40,50 & 74,00 \\
\hline 2 & 1200 & 1250 & 1020 & - & - & - & - \\
\hline 3 & 1000 & 1000 & 1030 & 87,55 & 17,00 & 36,00 & 74,00 \\
\hline 4 & 900 & 1000 & 990 & - & - & - & - \\
\hline 5 & 1000 & 780 & 1040 & 98,20 & 15,20 & 38,80 & 73,00 \\
\hline 6 & 800 & 700 & 1060 & 91,50 & 15,80 & 38,50 & 71,10 \\
\hline 7 & 800 & 710 & - & - & - & - & - \\
\hline 8 & 800 & 500 & 1040 & 95,00 & 16,00 & 35,85 & 74,00 \\
\hline 9 & 1850 & 1250 & 1065 & 91,00 & 15,40 & 37,00 & 67,90 \\
\hline 10 & 1800 & 1500 & 1085 & 97,60 & 17,90 & 36,20 & 67,20 \\
\hline 11 & 1600 & 2000 & 1060 & 97,00 & 16,00 & 36,80 & 74,00 \\
\hline 12 & 1850 & 1500 & 1020 & 92,30 & 17,80 & 34,10 & 74,70 \\
\hline 13 & 1750 & 1500 & 1115 & 86,90 & 15,50 & 40,90 & 74,70 \\
\hline 14 & - & - & - & - & - & - & - \\
\hline 15 & 2800 & 2500 & 1080 & 87,90 & 13,75 & 39,10 & 74,70 \\
\hline 16 & 2700 & 2250 & 1050 & - & - & - & - \\
\hline 17 & 2900 & 2000 & 1010 & 87,60 & - & 40,70 & 74,70 \\
\hline 18 & 2800 & 2500 & 1010 & 90,40 & 16,20 & 35,70 & 75,50 \\
\hline 19 & 3000 & 2520 & 1020 & 87,25 & 15,40 & 33,90 & 74,70 \\
\hline 20 & 2950 & 2000 & 1010 & 92,50 & 15,75 & 34,10 & 75,00 \\
\hline 21 & 2900 & 2500 & - & - & - & - & - \\
\hline 22 & 3100 & 2250 & 1005 & 93,50 & 16,00 & 35,00 & 74,50 \\
\hline $23 \mathrm{XII}$ & 2650 & 2500 & 990 & 92,50 & 15,50 & 34,50 & 75,00 \\
\hline
\end{tabular}

ingérée s'abaisse pour atteindre $1.000 \mathrm{~cm}^{3}$ au $12^{\mathrm{e}}$ jour de l'épreuve (soit : café $400 \mathrm{~cm}^{3}$, lait $100 \mathrm{~cm}^{3}$, bière $500 \mathrm{~cm}^{3}$ ), puis $500 \mathrm{~cm}^{3}$ (café $100 \mathrm{~cm}^{3}$, lait $100 \mathrm{~cm}^{3}$, bière $300 \mathrm{~cm}^{3}$ ), maintenue 5 jours de suite, puis seulement $400 \mathrm{~cm}^{3}$ (café $100 \mathrm{~cm}^{3}$, lait $100 \mathrm{~cm}^{3}$, bière $200 \mathrm{~cm}^{3}$ ), pendant 6 jours consécutifs.

Pendant le même temps, le poids de la nourrice, qui est de $72 \mathrm{~kg} .100$ au début, descend progressivement, avec quelques irrégularités, pour atteindre $69 \mathrm{~kg}$. 500 au $28^{\mathrm{e}}$ jour de la restriction hydrique, soit une perte de $2 \mathrm{~kg}$. 600 .

La courbe des urines descend de $1.580 \mathrm{~cm}^{3}$ à $480 \mathrm{~cm}^{3}$, la densité urinaire s'élevant à 1.026 , puis à 1.038 .

La production journalière de lait oscille entre des limites étroites, de $970 \mathrm{~cm}^{3}$ à $1.110 \mathrm{~cm}^{3}$, et se situe le plus souvent au-dessus de 
IIe EXPÉRIENCE

\begin{tabular}{|c|c|c|c|c|c|c|c|}
\hline 1) ates & $\begin{array}{c}\text { Boissons } \\
\text { ingérées } \\
\left(\mathrm{em}^{3}\right)\end{array}$ & $\begin{array}{c}\text { Diurèse } \\
\left(\mathrm{cm}^{3}\right)\end{array}$ & $\begin{array}{c}\text { Sécrétion } \\
\text { lactée }\left(\mathrm{cm}^{3}\right)\end{array}$ & $\begin{array}{c}\text { Extrait sec } \\
\text { dégraissé } \\
(\mathrm{gr} . \% o)\end{array}$ & $\begin{array}{l}\text { Protides } \\
\text { (gr. \%o) }\end{array}$ & $\begin{array}{l}\text { Lipides } \\
\text { (gr. \%o) }\end{array}$ & $\begin{array}{l}\text { Lactose } \\
\text { (gr. \%o ) }\end{array}$ \\
\hline $20 \mathrm{I}$ & - & - & 1060 & 96,8 & 17,5 & 41,00 & 74,0 \\
\hline 21 & - & - & 1040 & 101,5 & 18,5 & 39,9 & 70,6 \\
\hline 22 & 1500 & 1580 & 1120 & 98,2 & 17,0 & 41,0 & 72,0 \\
\hline 23 & 1400 & 1000 & 1030 & 94,7 & 16,6 & 38,2 & 71,8 \\
\hline 24 & 1900 & 1250 & 1040 & 92,7 & 17,2 & 37,2 & 70,4 \\
\hline 25 & 1100 & 1000 & 1060 & - & - & - & - \\
\hline 26 & 1400 & 1000 & 1020 & 90,2 & $\quad 18,5$ & 42,2 & 70,4 \\
\hline 27 & 1400 & 750 & 1060 & - & 16,4 & 40,1 & 71,0 \\
\hline 28 & 1300 & 750 & 1100 & 88,4 & 15,3 & 40,8 & 73,2 \\
\hline 29 & 1300 & 750 & 1050 & 88,9 & 16,8 & 40,5 & 74,0 \\
\hline 30 & 1020 & 720 & 1090 & 91,3 & 16,7 & 37,1 & 69,1 \\
\hline 31 & 900 & 520 & 1040 & 89,3 & 18,5 & 39,0 & 73,0 \\
\hline 1 II & 1000 & 750 & 1070 & - & - & - & - \\
\hline 2 & 900 & 750 & 970 & 90,4 & 18,7 & 41,5 & - \\
\hline 3 & 700 & 500 & 1010 & 90,8 & 18,7 & 39,7 & 69,0 \\
\hline 4 & 600 & 500 & 1110 & 94,9 & 17,7 & 38,2 & 69,0 \\
\hline 5 & 500 & 500 & 1080 & 92,5 & 16,5 & 38,9 & 67,8 \\
\hline 6 & 500 & 500 & 1020 & 88,9 & 15,1 & 38,5 & 67,0 \\
\hline 7 & 500 & 500 & 1070 & 92,4 & 19,8 & 35,9 & 67,8 \\
\hline 8 & 500 & 500 & 1100 & - & - & - & - \\
\hline 9 & 500 & 500 & 970 & 94,2 & 19,8 & 41,4 & 73,0 \\
\hline 10 & 400 & 480 & 1060 & 92,5 & 19,1 & 40,6 & 71,2 \\
\hline 11 & 400 & 480 & 970 & 93,5 & 17,8 & 37,1 & 70,4 \\
\hline 12 & 400 & 500 & 985 & - & 18,7 & 39,7 & 71,0 \\
\hline 13 & 400 & 480 & 990 & - & 18,8 & 41,1 & 73,0 \\
\hline 14 & 400 & 400 & 990 & 92,9 & 13,2 & 38,3 & 72,7 \\
\hline 15 & 400 & 480 & 1000 & - & - & - & - \\
\hline 16 & 550 & 500 & 990 & 95,9 & 18,0 & 38,5 & 67,8 \\
\hline 17 & 800 & 750 & 1010 & 90,8 & 18,5 & 41,9 & 68,5 \\
\hline 18 & 1500 & 750 & 1080 & 90,9 & 18,8 & 36,8 & 71,0 \\
\hline 19 & 1500 & 800 & 1040 & 87,3 & 15,8 & 37,3 & 67,0 \\
\hline 20 & 2000 & 1000 & 1020 & 92,0 & 17,3 & 39,5 & 67,8 \\
\hline 21 & 2100 & 1000 & 1025 & 84,4 & 17,3 & 41,6 & 68,5 \\
\hline 22 & 2200 & 1250 & 1040 & - & - & - & - \\
\hline 23 & 2000 & 1250 & 1000 & 90,4 & 18,1 & 41,8 & 69,1 \\
\hline 24 & 2000 & 1250 & 1050 & 91,6 & - & 39,2 & 74,0 \\
\hline 25 & 2500 & 1300 & 1010 & $88 ; 2$ & 17,7 & 39,8 & $69, \mathrm{i}$ \\
\hline 26 & 2600 & 1300 & 1975 & 89,0 & 18,2 & 40,1 & 74,0 \\
\hline 27 & 2500 & 1320 & 1030 & 90,7 & 16,1 & 39,6 & 74 \\
\hline 28 & 2600 & 1300 & 1090 & - & - & 41,9 & 74 \\
\hline 1 III & 2600 & 1500 & 1070 & - & - & - & - \\
\hline 2 & 2500 & 1500 & 1000 & - & - & 43,7 & 73,1 \\
\hline 3 & - & - & - & - & - & - & - \\
\hline 4 & 1520 & 1000 & 1030 & - & 16,4 & 44,7 & 71. \\
\hline 5 & 1500 & 780 & 960 & 91,5 & 17,9 & 42,9 & - \\
\hline 6 & 1500 & 780 & 1050 & - & - & - & - \\
\hline
\end{tabular}


$1.000 \mathrm{~cm}^{3}$. La composition du lait est pratiquement inchangée ; voici, à titre d'exémple, l'analyse du lait du dernier jour de l'épreuve:

$\begin{array}{lll} & \\ \text { Matières grasses } \ldots \ldots \ldots \ldots \ldots \ldots \ldots \ldots \ldots \ldots \ldots \ldots & 38,30 \\ \text { Lactose } \ldots \ldots \ldots \ldots \ldots \ldots \ldots \ldots \ldots \ldots \ldots \ldots \ldots \ldots & 72,75 \\ \text { Protides } \ldots \ldots \ldots \ldots \ldots \ldots \ldots \ldots \ldots \ldots \ldots \ldots \ldots & 13,20 \\ \text { Extrait sec dégraissé } \ldots \ldots \ldots \ldots \ldots \ldots \ldots \ldots \ldots \ldots & 92,90\end{array}$

- du 16 février au 6 mars (27e au $45^{\mathrm{e}}$ jour de l'expérience) : augmentation des boissons.

Après cette période de réduction, $\mathrm{M}^{\mathrm{me}} \mathrm{L}$. revient rapidement à une ration normale de liquides. Puis elle accepte de boire plus qu'à sa soif et pendant 11 jours elle ingère de 2.000 à $2.600 \mathrm{~cm}^{3}$ de liquides (lait, café, eau, bière).

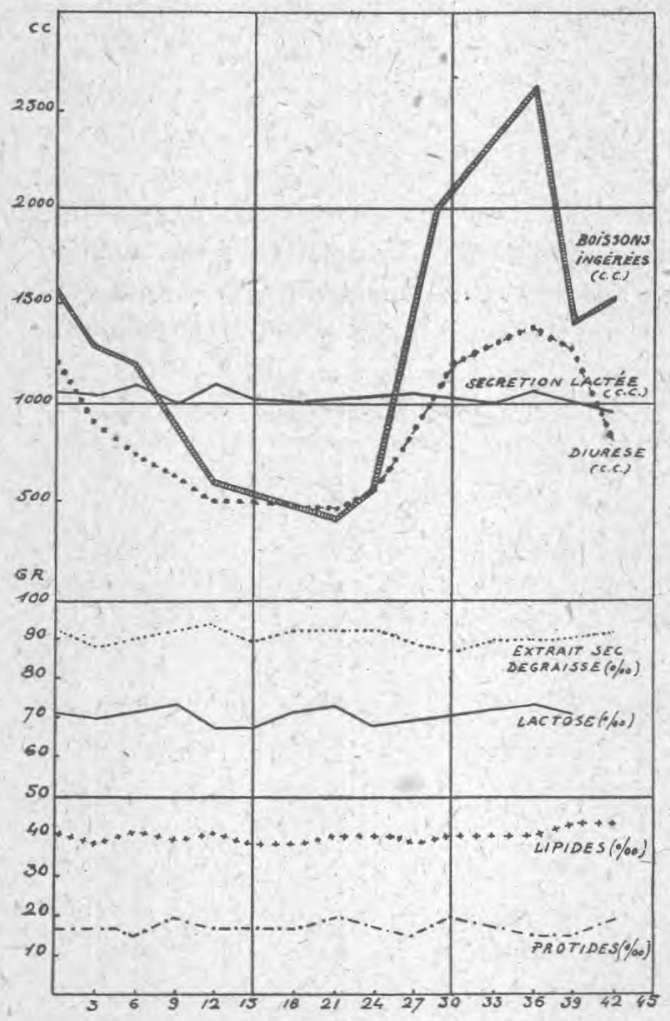

FIG 3

$2 \mathrm{e}$ expérience. - La composition du lait ne varie pas malgré les modifications de la quantité de boissons ingérées.

Les taux portés sur ce graphique représentent la eomposition moyenne du lait par périodes de 3 jours.

Pendant cette phase de l'expérience, le poids de la nourrice retrouve vite son niveau initial : $71 \mathrm{~kg} .900$.

La diurèse, partie de $500 \mathrm{~cm}^{3}$, remonte rapidement et décrit des oscillations entre $780 \mathrm{~cm}^{2}$ et $1.500 \mathrm{~cm}^{3}$ par jour. 
La production de lait reste entre 960 et $1.080 \mathrm{~cm}^{3}$ et, le dernier jour de l'épreuve, le lait a la composition suivante (toujours sur le mélange des 24 heures) :

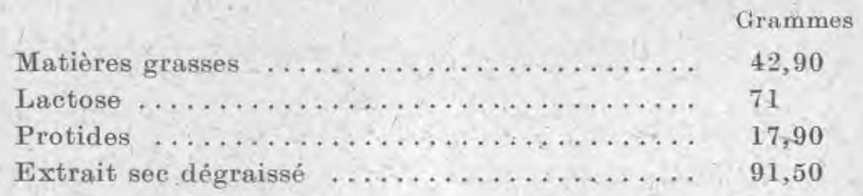

Ces deux expériences, réalisées avec le maximum de garanties, sont coneordantes et montrent clairement l'autonomie fonetionnelle, l'indépendance de la glande mammaire.

Pendant la restriction volontaire des boissons, le volume et la composition de la sécrétion lactée ne subissent aucun changement qu'on puisse imputer à cette restriction. Tant que la restriction est légère, seules les urines diminuent. Si la restriction est suffísamment poussée, la permanence du volume de la sécrétion et la constance de sa composition sont assurées, mais aux dépens des réserves de l'organisme et le poids corporel de la nourrice s'abaisse, conformément à une loi générale déjà bien connue (1). Nous ne pouvons dire ce qu'il serait advenu si nous avions franchi un degré de plus dans la restriction et atteint l'épuisement des réserves: lés conditions de notre expérimentation, malgré l'extrême bonne volonté de la nourrice qui nous prêtait son concours, ne nous autorisaient pas à aller jusque-là. Aussi conclurons-nous que, dans les limites de l'équilibre physiologique, la restriction des liquides est sans influence sur la sécrétion lactée établie. Signalons que dans des conditions très particulières, T. SALmI (2), au Lactarium de Helsinki, dans un pays fortement éprouvé par la guerre, a noté une baisse de la valeur calorique du lait récolté :-606 calories au litre en 1943, au lieu de 726 en 1939.

Quand l'apport liquidien excède les besoins physiologiques, les phénomènes inverses sont constatés. Les réserves d'eau de l'organisme bénéficient du supplément et le poids corporel s'élève. L'excédent, c'est-à-dire (quand l'organisme est en état d'équilibre), la plus grande part, est éliminé par les émonctoires naturels : reins, intestins, poumons, et peau. Là encore rien n'est changé au volume global et à la composition centésimale de la sécrétion lactée. La

(1) M $M^{\text {me }}$ Randoin, Rossier, Fournier et Mue Boisselot. Influence de la composition de la ration alimentaire actuelle sur la composition du lait maternel. Analyse in Revue de Diététique, 17.18 juin-juillet 1943, p. 204.

(2) T. Salmi. Composition et teneur en matières grasses du lait maternel en tenant compte des conditions alimentaires du temps de guerre. Acta Pcediatrica. Upsal, 32, I 30 septembre 1944, 1-26. 
"polylactie» (1), e'est-à-dire l'augmentation du volume de la sécrétion lactée par ingestion supplémentaire de liquide, n'existe pas plus dans l'espèce humaine que chez l'animal. Devant un régime riche en eau, la cellule mammaire reste indifférente : elle ne contribue pas à l'élimination de l'eau et ne saurait produire un lait " mouillé ».

La glande mammaire n'est pas un filtre pour l'eau du sang. Que l'eau du sang augmente ou diminue, la glande élabore une sécrétion à caractères constants pour un sujet donné, dont la teneur en eau (pour nous en tenir au point précis qui fait l'objet de cette étude) ne varie que dans des limites très étroites, indépendantes de la teneur en eau du régime alimentaire. Cette loi est valable tant que les réserves d'eau de l'organisme ne sont pas épuisées.

(Clinique de Puériculture de l'Hôpital Saint-Vincent-de-Paul.)

Ce travail a été effectué grâce à l'aide de la Fondation Arthur Sachs.

\title{
LE LAIT, SOURCE DE CONTAMINATION DE L'HOMIME ET DES ANIMAUX DANS LA TRANSMISSION DE LA FIĖVRE DU QUEENSLAND OBSERVÉE EN GRËCE (2)
}

\author{
par \\ M. JEAN CAMINOPETROS \\ Chef de Service à l'Institut Pasteur d'Athènes
}

Nous avons isolé en Grèce, en 1943, diverses souches de Rickettsias transmissibles en série au cobaye à partir du sang et des crachats de soldats allemands atteints de broncho-pneumonie.

Les troupes anglaises furent atteintes à leur tour, dès leur arrivée, en 1944.

En 1945, nous avons pu expédier une de ces souches aux Etats-Unis, où le Dr DingLe l'a identifiée avec Rickettsi Barneti, démontrant l'unité du virus de la grippe balkanique et de la fièvre du Queensland décrite en 1933.

Nous avons mené parallèlement les recherches expérimentales, les observations cliniques et épidémiologiques.

Les premières nous ont montré : la longue conservation du virus in vitro à la température ordinaire (jusqu'à deux ans), l'infection

(1) Ch. Porcher. La Polylactie. Annales des falsifications et fraudes, 1917, 105-106, p, 304 et La Sécrétion lactée, in Traité de Physiologie normale et pathologique, Roger et Binet, t. XI.

(2) Bulletin Aead. Méd., 1948, 132, 468. 